\title{
Voyage extraordinaire au centre du cerveau Jean-Didier Vincent
}

Jacques Epelbaum

Après Biologie des passions [1], désormais un classique, Jean-Didier Vincent nous propose un nouveau voyage à travers le cerveau : Voyage extraordinaire au centre du cerveau [2]. Le titre fait évidemment allusion à Jules Verne, mais il ne s'agit certainement pas de science fiction.

Comme tous les voyages, celui-ci commence par la consultation de la carte, autrement dit, l'anatomie du cerveau, le «paysage cérébral » du premier chapitre, puis vient le choix des «compagnons de voyage», une vingtaine de spécialistes, neurologues ou psychiatres, francophones, qui complètent chacun des chapitres par leurs connaissances précises. Méthodes d'investigation et problématiques de recherche sont quasiment identiques dans ces deux disciplines médicales qui n'en faisaient d'ailleurs qu'une jusqu'en 1968.

Le troisième chapitre est consacré à la météorologie, fluctuante comme l'état central, du contrôle de la température corporelle aux troubles de l'humeur, au gré des composantes circadiennes et saisonnières et de leurs traitements par les psychotropes.

En voyage, il est important de bien dormir, et Sigmund Freud et Michel Jouvet guideront le lecteur dans le sommeil et les rêves. Autres guides expérimentés, Jacques Faure, pionnier de

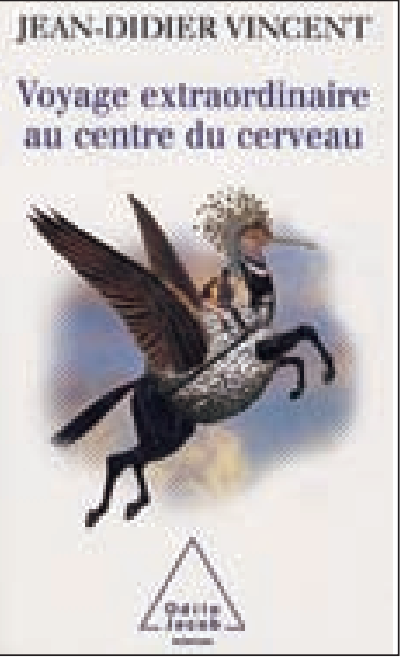
l'électroencéphalographie en France, ou Constantin Von Economo. On doit au premier la découverte du réflexe olfacto-bucco-ano-génito-sexuel chez la lapine, au second celle d'un schlafzentrum et d'un wachszentrum hypothalamiques chez les patients atteints d'encéphalite lors de l'épidémie de grippe espagnole qui envahit l'Europe en 1918. Les chapitres V à VIII explorent le manger et le boire, où l'auteur retrouve son complice le restaurateur Jean-Marie Amat (courez au Château du Prince Noir !), tous deux déjà associés dans une Nouvelle Physiologie du Goût [3]. Amateurs de rougets, lamproies, écrevisses, foie gras, pigeons, lièvres, obésité et diabète sucré vous guettent, pathologies dont

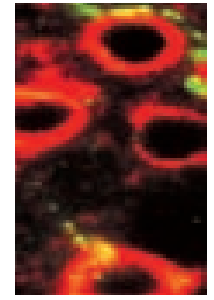

UMR 894 Inserm, Faculté de Médecine, Université Paris Descartes,

2ter, rue d'Alésia, 75014, Paris France. epelbaum@broca.inserm.fr

traitent les chapitres suivants, ainsi que de l'alcoolisme et de ses ravages. Transition naturelle avec les pages sur les lieux de plaisir du cerveau et le rire.

Il est temps maintenant de traiter des parties nobles du cerveau où s'élaborent les processus mnésiques. Je ne peux résister à citer l'introduction de ce chapitre sur le boulevard Pavlov: "On y croise les statues des héros qui ont contribué à la gloire du cerveau: le russe Ivan Pavlov (1849-1939) qui est représenté avec un chien; l'anglais Charles Scott Sherrington (1857-1952) qui montre du doigt un chat attaché sur une table de dissection; l'espagnol Santiago Ramon Y Cajal (1852-1934) assis devant un microscope; l'américain John Broadus Watson devant une usine et Eric Kandel (1929), le seul statufié de son vivant, dont on discerne avec difficulté ce qu'il tient dans sa main droite, une sorte de lièvre couché ou un morceau d'apfelstrudel?». Chacun aura reconnu la fameuse aplysie ou lièvre de mer ou, plus prosaïquement, les mollusques pisse-vinaigre du bassin d'Arcachon.

Les chemins de l'amour (déjà empruntés par l'auteur dans la Chair et le Diable) [4] nous mènent au salon des beaux-arts avec une description très précise des systèmes visuels et auditifs.

Après un hommage à Marcel Proust en guise d'exorde et sous l'égide de Georges Perec qui fut documentaliste au CNRS en Neurobiologie - on grimpe ensuite au grenier des souvenirs où s'exercent les différentes sortes de mémoire et leurs troubles associés. 
Dans le chapitre qui confronte la théorie de l'esprit au cerveau des facultés, c'est-à-dire l'isocortex, l'auteur rappelle l'inégalité de l'héritabilité du $\mathrm{Ql}$, et dans la présentation du cerveau moteur, il fait appel à Bernard Bouliac et Yves Agid, ce dernier décrivant combien les mouvements anormaux sont la «manifestation la plus spectaculaire, sinon la plus tragique d'un cerveau malade». Le dernier de ces chapitres est consacré au langage. En 70 ans, de ses premiers balbutiements à ses dernières paroles, un homme prononcera en moyenne 184800000 mots... Ceux que l'auteur a choisis dans ces 464 pages, très finement illustrées par François Durkheim, composent un livre remarquablement écrit, très documenté mais jamais ennuyeux, meilleur marché que Les 500 millions de la Begum, qui se lit en moins de temps qu'il n'en faut pour un Tour du monde en 80 jours ou même de Cinq semaines (en ballon), et qui n'a vraiment rien du Testament d'un excentrique. $\diamond$
Extraordinary journey in the center of the brain

\section{RÉFÉRENCES}

1. Vincent JD. Biologie des passions. Paris : Odile Jacob, 199 : 342 p.

2. Vincent JD. Voyage extraordinaire au centre du cerveau. Paris: Odile Jacob, $2007: 456$ p.

3. Amat JM, Vincent JD. Pour une nouvelle physiologie du goût. Paris: Odile Jacob, $2000: 244 p$.

4. Vincent JD. La chair et le diable. Paris: Odile Jacob, 2000 : 304 p.
TIRÉS À PART

J. Epelbaum

\section{Cycle de 3 conférences-débats :}

\section{L'embryon, le fœtus, l'enfant}

\section{Assistance Médicale à la Procréation (AMP) et lois de bioéthique}

L'embryon, le fœtus et l'enfant sont au cœur des lois de bioéthique, encadrant l'Assistance médicale à la Procréation, dont la révision est prévue en 2009.

L'Institut du droit de la famille et du patrimoine et l'Académie nationale de médecine se sont naturellement associés pour apporter dès à présent au débat leurs expériences respectives et leur réflexion.

Ces questions, outre qu'elles doivent faire l'objet d'échanges transdisciplinaires, ne peuvent plus rester l'apanage des seuls spécialistes. C'est pourquoi les participants aux conférences-débats seront largement associés à l'ensemble des discussions. Il s'agit de dépasser les frontières de la médecine et du droit pour permettre justement aux médecins et aux juristes de confronter leurs points de vue à la lumière de la philosophie, des sciences humaines et du débat public.

Ce cycle de conférences est organisé au cours de trois demi-journées, avec des interventions croisées de juristes, de médecins et de représentants des sciences humaines qui font référence sur ces sujets.

L'ensemble des interventions fera l'objet d'une publication par les éditions ESKA.

\section{Maison du barreau \\ 2-4 rue de Harlay, 75001 Paris}

Lundi 17 mars 2008 - L'embryon in vitro : qui ou quoi ?

LE CHAMP DES POSSIBLES : le pouvoir de procréer avec assistance médicale avec François THÉPOT, Frédérique DREIFUSS-NETTER, Jennifer MERCHANT

L’EMBRYON SUJET/OBJET DE SOINS \&T DE RECHERCHES avec Pierre JOUANNET, Grégoire LOISEAU, Simone BATEMAN

Jeudi 17 avril 2008 - Le fœtus dans tous ses états : quel statut ?

LE FGTUS : UN PATIENT ? avec Claude SUREAU, Agnès LOUIS-PECHA, Anne FAGOT-LARGEAULT

LA MATERNITÉ DE SUBSTITUTION avec Paul DEVROEY, Béatrice WEISS-GOUT, Dominique MEHL

Vendredi 16 mai 2008 - L'enfant issu d'une AMP : quelle filiation ?

NOUVEAUX MODES DE PROCRÉATION \&T ÉTABLISSEMENT D’UN LIEN DE FILIATION avec Bernard GOLSE, Brigitte FEUILLET-LE MINTIER, Carine CAMBY

PROCRÉATION MÉDICALISÉદ : Intervention de l’État et/ou dynamique de marché ? Bilan et perspectives

Synthèse de l'ensemble du cycle par Hervé CHNEIWEISS, Pierre MURAT et Alex MAURON

Clôture des travaux : Madame Valérie PECRESSE, Ministre de la Recherche et de l'Enseignement Supérieur

INFORMATIONS ET INSCRIPTION : CFE६ - Serge KEBABTCHIEFF - 12 rue du Quatre Septembre - 75002 Paris, Tél : 01428655 69/87 Fax : 0142604535 -congres@eska.fr 